\title{
Spinal ascendant necrosis with endotoxin shock associated to staphylococcus Aureus - case report
}

\author{
J osé Flores-Rivera ${ }^{1}$, Yamel Rito ${ }^{1}$, Katiuska Casares ${ }^{2}$, Martha L. Tena-Suck ${ }^{3}$ \\ 1. Service of Neurology, National Institute of Neurology and Neurosurgery. mexico city. Mexico. 2. Serice of Neuroimagen. \\ National Institute of Neurology and Neurosurgery. Mexico city. Mexico. 3. National Institute of Neurology and Neuro- \\ surgery, Mexico city, Mexico.
}

Correspondence: Martha L. Tena-Suck. Address: Department of Neuropathology, National Institute of Neurology and Neurosurgery, Mexico City, Mexico. E-mail: mltenasuck@gmail.com

Received: August 28, 2014

DOI : $10.5430 /$ crcp.v2n2p72
Accepted: January 26, 2015

URL: http://dx.doi.org/10.5430/crcp.v2n2p72

\section{Abstract}

Bacterial meningitis is rarely complicated by spinal cord involvement in adults. We report a We report a case of Staphylococcus aureus septicemia complicated by meningitis and extensive spinal cord injury, 21-year-old man with tattoos all over the body. The patient's neurological condition worsened within two days, leading to ascending brain stem necrosis and death. The magnetic resonance imaging (MRI) demonstrated intramedullary hyperintensity on T2-weighted image. Postmortem microscopic examination confirmed that the extensive spinal cord injury was of ischemic origin, caused by diffuse leptomeningitis and endarteritis and multiple pulmonary micro-abscess and intravascular disseminated disease and neurotoxic shock associated to Staphylococcus Aureus was diagnosed.

\section{Key words}

Autopsy case, Bacterial meningitis, Immunohistochemistry, Sepsis, Spinal cord injury

\section{Introduction}

Myelitis is defined as inflammatory disease of the spinal cord irrespective of the underlying aetiology or pathologicanatomic alterations. Direct infections, post infectious, post vaccinal immunological processes, or other (auto) immunological diseases such as multiple sclerosis or systemic vasculitis. The spinal cord may be injured by compression due to a spinal abscess, by ischemia due to vasculitis, shock, herniation, arachnoiditis, or by myelitis.

Bacterial meningitis is rarely complicated by acute spinal cord involvement and (e.g. myelitis, ischemic infarction, spinal abscess, or epidural hemorrhage) ${ }^{[1,2]}$. In spinal cord dysfunction, magnetic resonance imaging (MRI) is the imaging modality of choice. MRI findings of myelitis due to bacterial meningitis in adults have been reported ${ }^{[1-5]}$.

The immunologically suppressed patient spilling from viral infections (especially herpes simplex, varicella-zoster virus, cytomegalovirus), bacterinal infections (tuberculosis), but rarely from parasitic infections ${ }^{[2]}$. The immunologically competent patient could suffer from bacterial (borreliosis), but rarely viral infections, sarcoidosis and demyelinating diseases, and idiopathic myelitis ${ }^{[1-5]}$. 
Secondary ischemic and demyelinating processes result in a complex morphology of inflammatory lesions on MRI, and therefore the whole spectrum of demyelinating, ischemic and inflammatory lesions has to be included in the differential diagnosis ${ }^{[3]}$. Even tumors may imitate inflammatory myelitis and radiculitis ${ }^{[1-6]}$. Most commonly, meningoradiculitis can be separated from myelitis. A reliable diagnosis of a specific inflammatory lesion is difficult and is mostly accordingly in patients with multiple sclerosis and in patients with HIV-associated cytomegalovirus infection ${ }^{[6,7]}$.

We describe a fatal case of S. Aureus septicemia complicated by meningitis and extensive spinal cord injury, leading to ascending brain stem necrosis. Repeated MRI and a postmortem examination were performed.

\section{Clinical case}

A 21-year-old male who had a history of multiples tattoos spilling the last one made few days before his hospitalization. He with AIDS sero-negative. Five days later he presented paresthesia and pain in soles. This pain was ascendant, with climbed, distal tremor addition, dysuria, polyuria, weakness in both lower limbs predominantly right side, dysphonia, and dysarthria, dyspnea, agitation, inattention, with sagging hypotonic widespread and affection of cranial nerves XI, X, XI and XII, force $3 / 5$ overall. The physical examination showed diaphoresis, piloerection and uncontrolled sphincters and increased sensory reflexes and the nerve conduction velocity showed block conduction and he was admitted at our institution and one day after he dead by severe rostrocaudal deterioration and coma The admission laboratory samples revealed a major inflammatory syndrome (C-reactive protein level of $47.8 \mathrm{mg} / \mathrm{dl}$; normal range $<1 \mathrm{mg} / \mathrm{dl}$ ), with a normal white blood cell count. In addition, renal function was mildly impaired, serum creatinine level was $3.1 \mathrm{mg} / \mathrm{dl}$ (normal range $0.6 \mathrm{mg} / \mathrm{dl}-1.4 \mathrm{mg} / \mathrm{dl}$ ) and blood urea nitrogen was $76 \mathrm{mg} / \mathrm{dl}$ (normal range $15 \mathrm{mg} / \mathrm{dl}-50 \mathrm{mg} / \mathrm{dl}$ ). There was no evidence of a pulmonary or urinary infection. However, an empirical antimicrobial therapy with amoxicillin and clavulanic acid and steroids, and plasmapheresis was initiated. Lumbar puncture revealed a purulent cerebrospinal fluid (CSF). The white blood cell count was $31,800 / \mathrm{mm}^{3}$ (normal range $<5,000 / \mathrm{mm}^{3}$ ) with $85 \%$ neutrophils, $10 \%$ lymphocytes and $5 \%$ monocytes. The CSF glucose was $44 \mathrm{mg} / \mathrm{dl}$ and lactate $12.5 \mathrm{mmol} / \mathrm{L}$ (normal range $1 \mathrm{mg} / \mathrm{dl}-2.2 \mathrm{mg} / \mathrm{dl}$ ). Simultaneous blood glucose determination was not available. The CSF cultures grew S. Aureus.
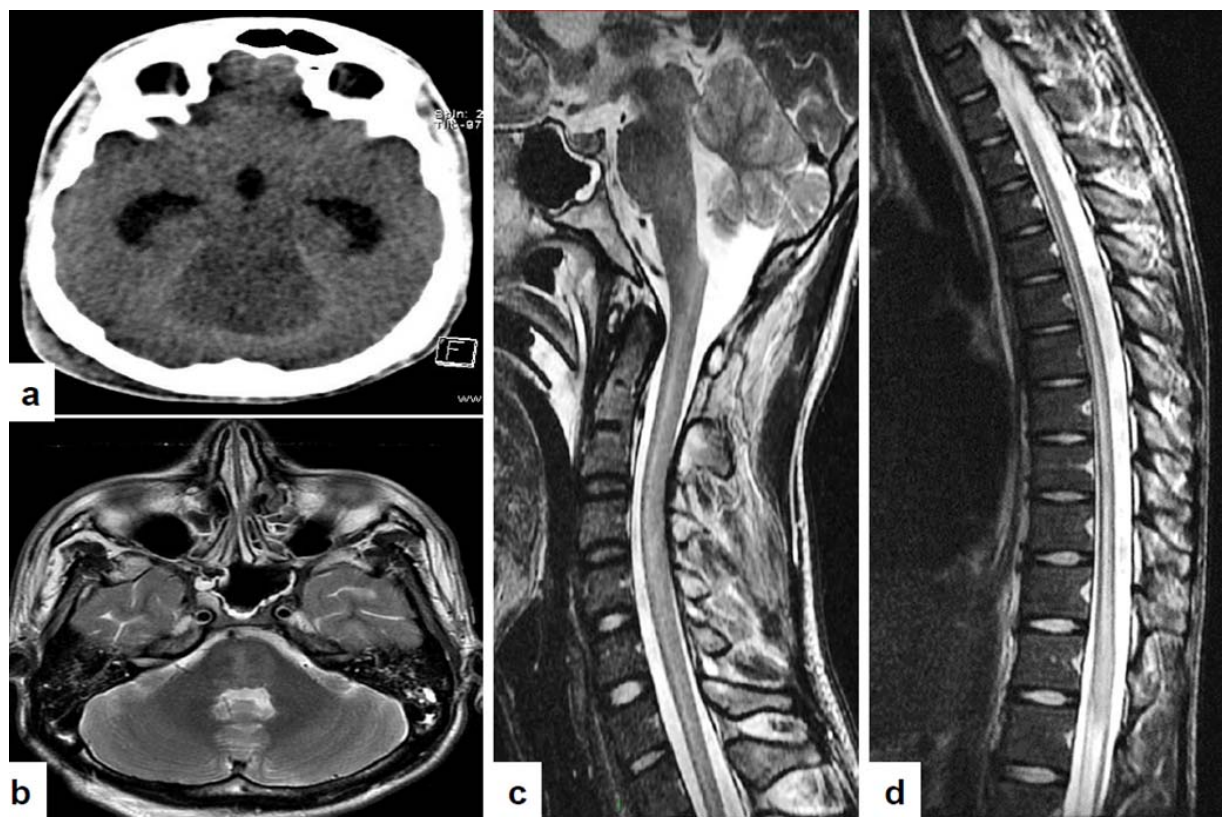

Figure 1. (a) Single phase CT scan shows hypodensity in the midbrain edema and ventricular dilatation, with cerebellar enhancement was observed. (b) MRI axial T2 with cerebellar vermix and pons hyperintensity. (c) Cervical and dorsal T2 Sagittal MRI with intramedullary hyperintensity from $\mathrm{C} 1$ to conus. (d) Also observed hyperintensity in midbrain, pons and bulb. 


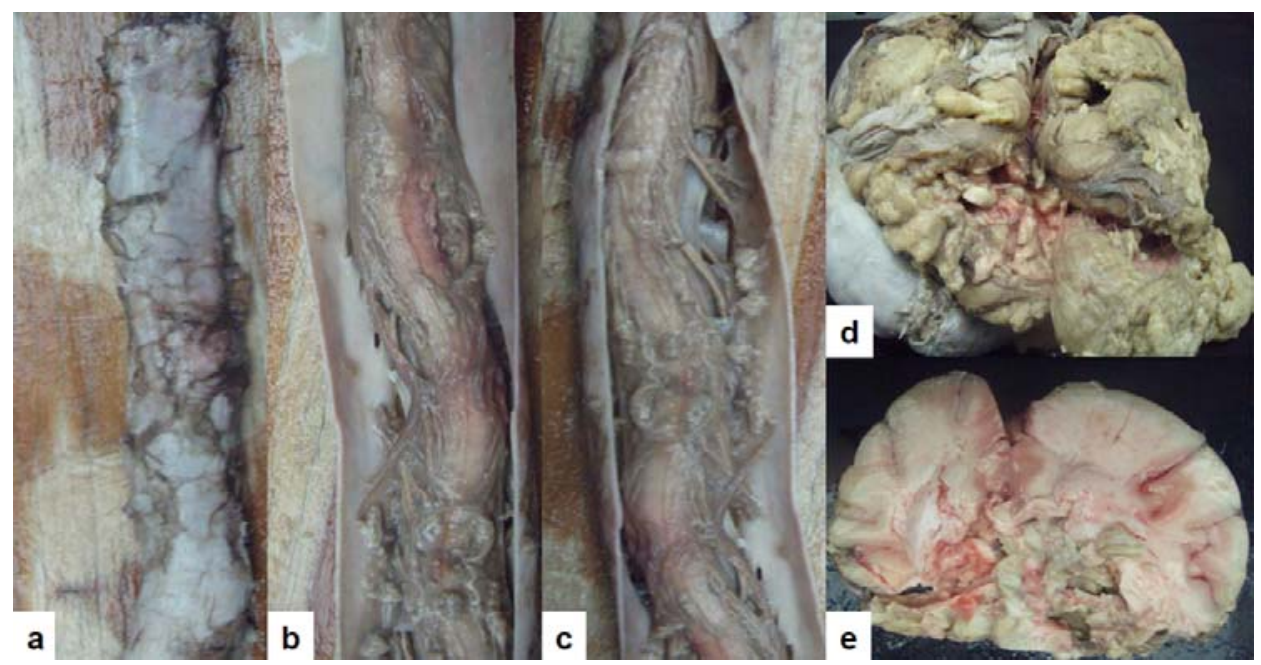

Figure 2. Macroscopically appearance of spinal cord. (a) The Spinal cord meninges observed inflammed, bulging, foamy, unpolished overshadowed and hemorrhagic aspect (b). To cut out was observed a chocolate-looking like, (c) necrotic material bleeding. (d) The encephalon showed that the infratentorial area with necrosis, hemorrhage and soft appearance, easily fall part. Cerebellum was also necrotic. (e) Coronal cuts showed liquefactive necrosis in midbrain.

The MRI showed non-communicating hydrocephalus requiring ventricle peritoneal shunt (see Figures 1a and 1b). The spinal MRI showed spinal cord injury in T2 (see Figure 1c), it was heterogeneous patches that affecting pontine and vulvar region, as well as cerebellum and basal nucleus (see Figure 1d). The patient died from cardiocirculatory failure. Complete autopsy was performed. In the gross examination; Spinal cord meninges were inflamed, bulging, unpolished overshadowed and hemorrhagic aspect (see Figure 2a). To open it, showed a chocolate-looking necrotic material (see Figures $2 \mathrm{~b}$ and $2 \mathrm{c}$ ). The encephalon with 1.300 grs weight, showed that the infratentorial areas were necrotic, hemorrhagic and soft, easily fall part. Cerebellum was also necrotic (see Figure 2c). Coronal cuts showed liquefactive necrosis in midbrain (see Figure 2d).
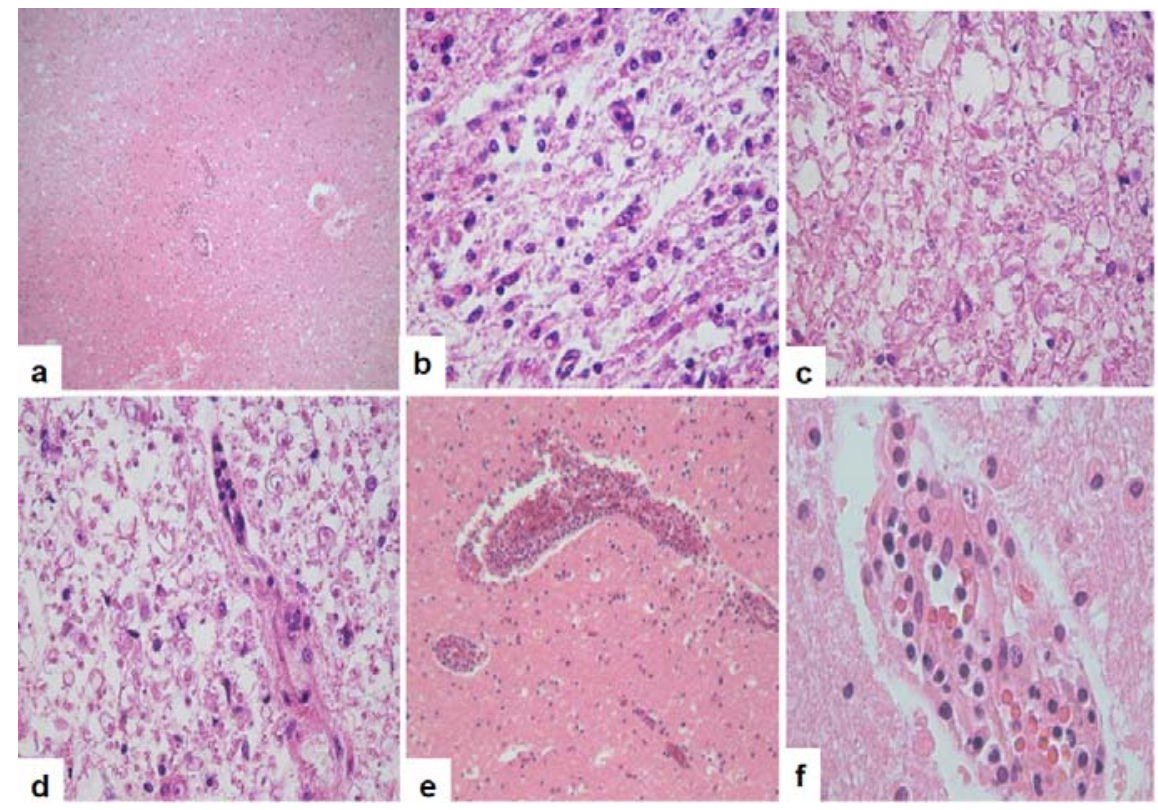

Figure 3. Histological examination of the spinal cord that in (a) showed leptomeningitis with vasculitis and secondary medullar severe ischemia, (b) showed varied degrees of necrosis and cellular detritus, (c) fibrin and some perivascular inflammatory cells were observed. (d) Perivascular lymphocytes observed. (e) Mesencephalon showed isquemic necrosis with vascularity's in (f). (H\&E 400×). 
Histological examination spinal cord showed leptomeningitis with vasculitis and secondary medullar sever ischemia (see Figure 3a), and varied grade of necrosis (see Figures $3 \mathrm{~b}$ and 3c), cellular detritus, fibrin and some perivascular inflammatory cells were observed (see Figure 3d) and lymphocytes into the nerves fibers were observed Bulb, pons, mesencephalon, cerebellum and hypothalamus showed isquemic necrosis (see Figure 3e) with vascularites (see Figure 3f). Frontal cortex was the most preserved area. However, neuronal isquemic injury was observed. Pulmonary thrombosis as well micro-abscesses were observed. Candidiasis in trachea and bronchus was observed. Immunohistoquemistry was performed. And figures are seen in Figure 4. GFAP was weak and scarce astrocyte positive in spinal cord, cerebrum and in cortical fontal cortex. Myelin was weak and vacuolated aspect in spinal cord, and demyelinating areas were observed in white matter. Perivascular inflammatory cells were immunoreaction positive to CD56, IL2 and IL6, CD68, TNF $\alpha$, IL10, CD4, CD38 and were negative to CD8, IL12, MHC-II. Citomegatovirus, Herpesvirus 1 and herpesvirus 2 were also negative. In basic of these findings shock endotoxin septic syndrome was diagnosed as cause of dead. The CSF culture positive to Staphylococcus Aureus catalase positive was essential to make this diagnosis.
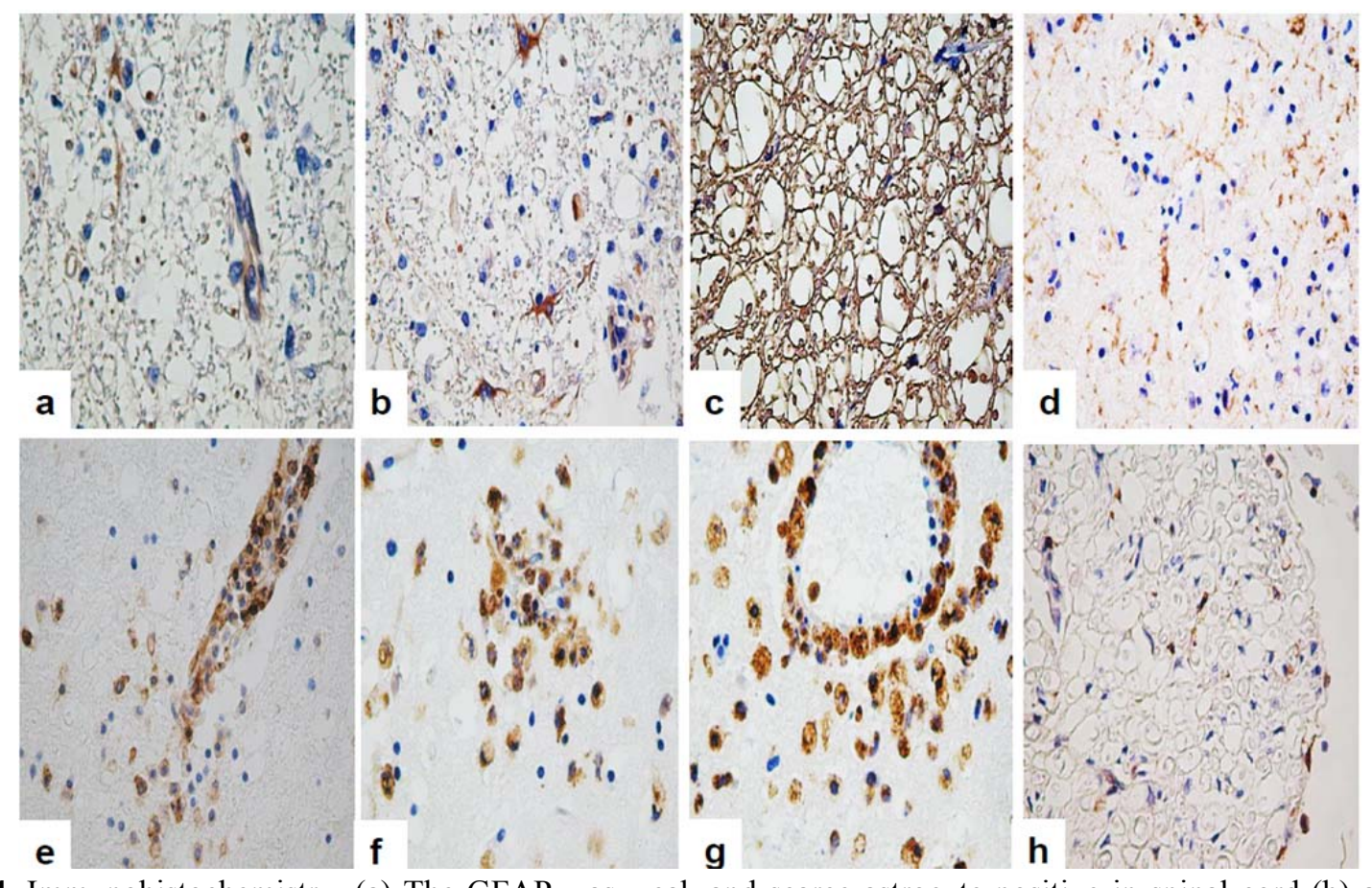

Figure 4. Immunohistochemistry. (a) The GFAP was weak and scarce astrocyte positive in spinal cord (b) and (b) in cerebellum. (c) Mylin immunoreaction was weak in spinal cord, and weak demyelination areas observed in white substance (d). (e) Perivascular inflammatory cells were immunoreaction positive to IL6 positive cells, (f) CD68 positive cells, (g) Positive TNF $\alpha$ cells and the peripheral nerves showed occasional CD4 positive lymphocytes (Original magnification $400 \times$ ).

\section{Discussion}

Bacterial meningitis is rarely complicated by spinal cord involvement, children are more frequently affected than adults ${ }^{[1-7]}$. The spinal cord may be injured by compression due to a spinal abscess, by ischemia due to vasculitis, shock, herniation, arachnoiditis, or by myelitis and fournier gangrene in spinal cord injury have been reported ${ }^{[6,7]}$. The causative germs are mainly Neisseria meningitidis, Streptococcus pneumoniae, Escherichia coli, and Haemophilus influenzae, while the involvement of $S$. aureus seems exceptional ${ }^{[1-5]}$. Our patient initially presented signs of spinal cord injury with quadriparesis, and meningitis, rapidly progressive manifestations of brain stem involvement (dysarthria and respiratory failure). De Schryver N et al. ${ }^{[3]}$ reported a similar case with extensive spinal cord injury following S. Aureus septisemia ${ }^{[7-9]}$. 
The prevalence of acute transverse myelitis associated with S. aureus is rare. Most of the time, it occurs as a consequence of a hematologic spread from another infected site that can be pulmonary, cardiac, skeletal, genitourinary, gastrointestinal or cutaneous ${ }^{[3]}$. S. aureus myelitis may also develop from a local infection such as a pyomyositis of the paraspinal muscles ${ }^{[3]}$. Acute myelopathy includes a group of diseases with an important associated morbidity. The most frequent etiology is extramedullary compression ${ }^{[3]}$. The differential diagnosis among intramedullary abscess, epidural abscess, acute transverse myelitis and spondylodiscitis associated to acute myelopathy rare case of necrosis associated to $S$. aureus have been reported. And differential diagnosis of non-traumatic, acute transverse spinal cord syndromes should cover compressive myelopathy (mostly hematomas or tumors), inflammatory myelitis and vascular myelopathies ${ }^{[3,7-9]}$. Since acute pathologies of the spinal cord primarily result in flaccid para- or tetraparesis accompanied by areflexia or hyporeflexia (spinal shock), acute polyradiculoneuritis and the caudal equine syndrome must also be weighed into the differential diagnosis ${ }^{[3]}$. Paraplegia may ultimately also be of psychogenic origin ${ }^{[3,7-9]}$. The clinical picture is characterized by the rapidity of progression, the possible involvement of pain, and the specific pattern of the deficits ${ }^{[3]}$. When the latter occurs, localization of the rostrocaudal level and transverse spread are crucial factors. Depending on the affected structure, one differentiates between anterior spinal cord syndromes (anterior spinal artery syndrome, selective involvement of the anterior horn, centromedullary syndromes) ${ }^{[1-6]}$. The diagnosis of an infectious myelitis is based on MRI findings, which typically show a spinal cord hypersignal on T2-weighted images, which is sometimes associated with swelling ${ }^{[3]}$. The differential diagnosis of an intramedullary spinal cord lesion with a high T2 signal intensity on MRI should also include primary neoplasm and infarction involving the anterior spinal artery ${ }^{[7-9]}$.

Toxic shock syndrome (TSS) is an acute toxin-mediated infectious syndrome characterized by fever, hypotension, desquamation, and multiorgan involvement. It is a rare condition (incidence of $0.79 / 100,000$ women), particularly in the adolescent population, and it may be menstrual (mTSS) or non-menstrual (nmTSS) in origin ${ }^{[7]}$. Rare cases associated with spinal necrosis have been reported. This is one of them with this rare and acute quietly progressive disease. S. aureus produces a wide variety of exoproteins ${ }^{[7-9]}$. The main function of these proteins may be to convert local host tissues into nutrients required for bacterial growth. Some strains produce one or more additional exoproteins, which include toxic shock syndrome toxin-1 (TSST-1) ${ }^{[7-9]}$, etc. Each of these toxins is known to have potent effects on cells of the immune system, but many of them have other biological effects as well. The high frequency of IFN- $\gamma^{+}, \mathrm{TNF}-\alpha^{+}$, and IL- $2^{+}$, HTLV-1-specific $\mathrm{CD}^{+}{ }^{+}$cells raises the possibility that such cells contribute to the pathogenesis of inflammatory CNS disease by forming self-perpetuating inflammatory lesions. The neurotoxicity of alpha-toxin has been observed in the destruction of myelin sheaths surrounding nerves and in the cerebral cortex. An effect on macrophages has also been observed, resulting in increased secretion of interleukin- $1 \beta$. These widespread effects, and possibly many others, are due primarily to the alteration of the ion balance within the host. The breadth of the effect is consistent with the ability of the toxin to interact with specific receptors on the cell surface as well as nonspecifically with the membrane. The clinical progression correlated with the worsening of the MRI findings and the electrophysiological testing. Indeed, deterioration of this disease indicated structural pontine damage, and severe damage to the lemniscal pathways caudal to the bulbo-medullary junction necrosis ${ }^{[3,7-9]}$.

In conclusion we report a rare case of TSS with ascendant spinal necrosis in a young man after tattoo procedures. This one corresponded to the second case report.

\section{References}

[1] Kastenbauer S, Winkler F, Fesl G, Schiel X, Ostermann H, Yousry TA, et al. Acute severe spinal cord dysfunction in bacterial meningitis in adults: MRI findings suggest extensive myelitis. Arch Neurol. 2001; 58: 806-810. PMid: 11346376. http://dx.doi.org/10.1001/archneur.58.5.806

[2] Moffett KS, Berkowitz FE. Quadriplegia complicating Escherichia coli meningitis in a newborn infant: case report and review of 22 cases of spinal cord dysfunction in patients with acute bacterial meningitis. Clin Infect Dis. 1997; 25: $211-214$. http://dx.doi.org/10.1086/514534

[3] De Schryver N, Cosnard G, Van Pesch V, Godfraind C, Hantson P. Extensive Spinal Cord Injury following Staphylococcus aureus septisemia and meningitis. Case report Neurol. 2011; 3: 147-153. PMid: 21738506. http://dx.doi.org/10.1159/000329841 
[4] Kikuchi M, Nagao K, Muraosa Y, Ohnuma S, Hoshino H. Cauda equina syndrome complicating pneumococcal meningitis. Pediatr Neurol. 1999; 20: 152-154. http://dx.doi.org/10.1016/S0887-8994(98)00129-5

[5] O'Farrell R, Thornton J, Brennan P, Brett F, Cunningham AJ. Spinal cord infarction and tetraplegia, rare complications of meningococcal meningitis. Br J Anaesth. 2000; 84: 514-517. PMid: 10823108.

http://dx.doi.org/10.1093/oxfordjournals.bja.a013482

[6] Puvabanditsin S, Wojdylo EW, Garrow E, Kalavantavanich K. Group B streptococcal meningitis: a case of transverse myelitis with spinal cord and posterior fossa cysts. Pediatr Radiol. 1997; 27: 317-318. PMid: 9094237. http://dx.doi.org/10.1007/s002470050137

[7] Mathew P, Todd NV, Hadley DM, Adams JH. Spinal cord infarction following meningitis. Br J Neurosurg. $1993 ; 7$ : $701-704$. PMid: 8161436. http://dx.doi.org/10.3109/02688699308995103

[8] Friess HM, Wasenko JJ. MR of staphylococcal myelitis of the cervical spinal cord. AJNR Am J Neuroradiol. 1997; $18: 455-458$. PMid: 9090402.

[9] Kulkarni GB, Pal VK, Veena Kumari HB, Goyal M, Kovoor JM, Nadig S, et al. Community-acquired methicillin-resistant Staphylococcus aureus pyomyositis with myelitis: a rare occurrence with diverse presentation. Neurol India. 2009; 57: 653-656. PMid: 19934571. http://dx.doi.org/10.4103/0028-3886.57809 